\title{
DIAGNÓSTICOS RADIOGRÁFICOS DE CANINOS E FELINOS COM HIPERPARATIREOIDISMO NUTRICIONAL SECUNDÁRIO
}

\author{
DOMINGOS JOSÉ STURION1 \\ PATRÍCIA MENDES PEREIRA 2
}

STURION, Domingos José; PEREIRA, Patrícia Mendes. Diagnósticos radiográficos de caninos e felinos com hiperparatireoidismo nutricional secundário. Semina: Ci. Agr., Londrina, v. 16, n. 1, p. 28-33, mar. 1995.

\begin{abstract}
RESUMO: O objetivo deste trabalho foi analisar os laudos radiográficos executados, no Hospital Veterinário da Universidade Estadual de Londrina no perlodo de 1978 a 1993 com a finalidade de levantar a casulstica de hiperparatireoidismo nutricional secundário, procurando observar a distribuição quanto ao sexo, idade, raça, estado de nutrição e tipo de alimentação. Foram encontrados 136 felinos e 81 caninos de diferentes raças, sendo maior a porcentagem do sexo masculino. A faixa etária mais acometida em ambas espécies esteve entre 0 a 6 meses. Na análise das raças dos caninos verificou-se uma grande porcentagem de animais de grande porte. $O$ estado de nutrição aparente verificado foi bom para maioria dos animais. A alimentação destes animais eram deficientes em Ca ou rica em F (Ex: fuba), levando a uma dieta desbalanceada.
\end{abstract}

PALAVRAS-CHAVE: Hiperparatireoidismo nutricional secundário; cães; alimentação.

\section{INTRODUÇĀO}

As necessidades nutricionais, a alimentação e os cuidados com cães e gatos durante a fase de crescimento devem ser diferenciados em relação aos animais adultos. Assim dietas pobres em cálcio ou excessivamente ricas em fósforos, ou com menor disponibilidade de cálcio devido a insuficientes niveis de vitamina $D$ ou $A$, são fatores que levam ao desenvolvimento de osteodistrofia mais frequente nas espécies canina e felina, denominada de hiperparatireoidismo nutricional secundário (DOIGE, 1990).

O hiperparatireoidismo nutricional secundário é uma doença metabólica que ataca tanto animais domésticos quanto silvestres, e é caracterizada por uma secreção compensatória aumentada de paratormônio, devido a uma dieta desbalanceada na relação cálcio:fósforo (Ca:P) (GODDARD et al., 1970; PRICE, 1970; WATSON, 1981).

SHERWOOD et al. (1968) afirmam que rações com baixos teores de cálcio, resultaram numa hipocalcemia associada a um aumento da paratireóide. HUBBARD et al. (1979) observaram que após 259 dias, cães com dietas pobres em cálcio apresentaram sinais de hiperparatireoidismo nutricional secundário.

Como informaram KROCK et al., (1963), a doença está relacionada com a ingestão de carne na alimentação por um tempo prolongado. Já BRASS et al. (1975). constataram que o problema também é devido a uma alimentação rica em fósforo. Pois, segundo DOIGE
(1990), o alto aporte de fósforo resulta em hiperfosfatemia transitória. Embora a concentração sanguinea de fósforo não exerça influência reguladora direta sobre a síntese e secreção do paratormônio, esta concentração pode, quando elevada, contribuir secundariamente para a estimulação da paratireóide em virtude da sua capacidade de reduzir os niveis sanguíneos de cálcio.

Segundo GODARD et al. (1970), o hiperparatireoidismo nutricional secundário ocorreu rapidamente em gatos quando alimentados com carne de coração, rim ou fígado, que tem menos de $10 \mathrm{mg}$ de cálcio por $100 \mathrm{~g}$ de carne e uma relação Ca:P de 1:20 (coração) e 1:50 (fígado e rim).

SCOTT (1965) salientou que um gato em crescimento requer de 200 a $400 \mathrm{mg}$ de cálcio por dia e 150 a $400 \mathrm{mg}$ de Fósforo por dia. Jå MILLER (1969), descreveu que a reabsorção óssea mediada pelo paratormônio e a substituição por tecido fibroso resultou numa diminuição do volume ósseo e doença generalizada do esqueleto caracterizada por osteodistrofia fibrosa. A falta da integridade do esqueleto em gatos jovens pode resultar em deformidades como xifoses, escolioses e lordoses. O que para BENNETT (1976), isto se deve a substituição do tecido b́sseo por tecido fibroso contendo trabéculas muito finas ou osso imaturo, causando distúrbios nà epífise em crescimento com resultante deformidade óssea.

Quando há aumento no paratormónio circulante, este age nos rins inibindo a reabsorçäo tubular de fosfato e diminuindo a perda de cálcio, para que o nivel sangul-

1 - Professor Dr. Adjunto do Departamento de Medicina Veterinária da UFPR, Curitiba, Paraná, Brasil.

2 - Acadêmica de Medicina Veterinária da Universidade Estadual de Londrina, Londrina, Paraná, Brasil.

Semina Cl. Agr., v. 16, n. 1, p. 28-33 
neo volte ao normal. A ação do paratormônio nos ossos é caracterizada por um aumento na reabsorção que leva a nível de Ca para o normal. O aumento da atividade dos osteoclastos é generalizada, mas há sinais de lugares de predileção onde a doença se desenvolve com mais intensidade como: na espécie felina, particularmente nos ossos da crista interdental, seguido por ossos do crânio, vértebras, áreas metafisarias e cortical de ossos longos (ROWLAND \& FETTER, HAZEWINKEL, 1989).

De acordo com BRASS et al. (1975), a patologia foi constatada principalmente em cães de raças de grande porte e entre as idades de 3 a 12 semanas de vida.

ROWLAND et al. (1968) observaram que gatos jovens ficaram menos ativos, relutantes em se movimentarem e desenvolveram claudicação de posteriores e ataxia, após consumirem carne de coração por 4 a 8 semanas.

Para GODDARD et al. (1970) e BENNETT (1976), os sinais clínicos mais comuns em cães eram: claudicação, fraturas compressivas de ossos longos, excessiva dorsofleção do carpo e tarso; anorexia e dispneia. No diagnóstico, para os autores são importantes: a consistência dos ossos, a radiografia do esqueleto e a determinação de cálcio e fósforo.

HOSKINS (1990) descreveu a perda da densidade óssea. A zona de crescimento aparece normal, e há um aumento da radiodensidade na metáfise, o que provavelmente representa a área de mineralização preferencial óssea.

Os problemas causados pelo hiperparatireoidismo nutricional secundário (HNS) e a sua grande casuística na região de Londrina (PR) permitiram a realização deste trabalho, cujo objetivo foi observar a incidência do HNS nas espécies canina e felina, em relação a raça, idade, tipo de alimentação e estado nutricional mais frequentes.

\section{MATERIAL E MÉTODO}

Foram realizados levantamentos dos laudos radiográficos executados no Hospital Veterinário da UEL, no período dę 1979 a 1993, com a finalidade de observação da caracteristica do hiperparatireoidismo nutricional secundário em caninos e felinos, procurando observar a distribuição quanto ao sexo, idade, raça, estado de nutrição e tipo de alimentação.

Foram levantados os dados de 81 cães, sendo que destes, 33 eram sem raça definitiva, 27 Pastores Alemães, 8 Dobermans, 2 Filas Brasileiros, 2 Boxes, 2 Dogs Alemães, 2 Basser Hounds, 1 São Bernardo, 1 Cocker Spaniel, 1 Akita, 1 Dachshund, 1 Setter e 136 felinos, sendo que destes 73 eram sem raça definida, 57 siameses, 4 jaguatiricas, 1 Angorá e 1 Persa.

Do total de cães, 48 eram machos e 33 fêmeas, e do total de felinos, 69 eram machos, 65 fêmeas e 2 não foram identificados.

\section{RESULTADOS}

Os dados obtidos, relacionados aos caninos foram distribuidos nas Tabelas de 1 a 5.

TABELA 1 - Distribuiçāo dos sexos dos caninos com hiperparatireoidismo secundário (HNS) no periodo de 1979 a 1993 no Hospital Veterinårio (HV) da Universidade Estadual de Londrina (UEL)

\begin{tabular}{lcc}
\hline SEXO & No DE CASOS & $\%$ \\
\hline Macho & 48 & 59,250 \\
Fêmea & 33 & 40,741 \\
\hline
\end{tabular}

TABELA 2 - Distribuição das idades mais frequentes dos caninos com HNS no periodo de 1979 a 1993 no Hospital Veterimário (HV) da Universidade Estadual de Londrina (UEL)

\begin{tabular}{lcl}
\hline IDADE (Meses) & No DE CASOS & $\%$ \\
\hline $0-3$ & 33 & 40,741 \\
$3-6$ & 37 & 45,679 \\
$6-9$ & 4 & 4,9 \\
$9-12$ & 2 & 2,46 \\
$12-15$ & 3 & 3,7 \\
Sem registro & 2 & 2,46 \\
\hline TOTAL & 81 & 100,00 \\
\hline
\end{tabular}

TABELA 3 - Distribuiçāo das raças dos caninos com HNS no periodo de 1979 a 1993 no Hospital Veterinårio (HV) da Universidade Estadual de Londrina (UEL)

\begin{tabular}{lcc}
\hline RAÇA & No DE CASOS & $\%$ \\
\hline SRD & 33 & 40,7 \\
Pastor Alemão & 27 & 33,3 \\
Doberman & 8 & 9,87 \\
Basset Hound & 2 & 2,46 \\
Boxer & 2 & 2,46 \\
Dog Alemão & 2 & 2,46 \\
Fila Brasileiro & 2 & 2,46 \\
Akita & 1 & 1,235 \\
Cocker Spaniel & 1 & 1,235 \\
Dachshund & 1 & 1,235 \\
\hline TOTAL & 81 & 100,00 \\
\hline
\end{tabular}


TABELA 4 - Distrỉbuição dos tipos de alimentạ̧āo dos caninos com HNS no período de 1979 a 1993 no Hospital Veterinårio (HV) da Universidade Estadual de Londrina (UEL)

\begin{tabular}{lcc}
\hline TIPO DE ALIMENTAÇÃO & No CASOS & $\%$ \\
\hline Sem registro & 40 & 49,38 \\
Fubá & 13 & 16,04 \\
Comida caseira & 19 & 23,45 \\
Ração & 5 & 6,17 \\
Leite & 1 & 1,235 \\
Carne & 3 & 3,704 \\
\hline TOTAL & 81 & 100,00 \\
\hline
\end{tabular}

TABELA 5 - Distribuição do estado de nutrição dos caninos com HNS no periodo de 1979 a 1993 no Hospital Veterinário (HV) da Universidade Estadual de Londrina (UEL)

\begin{tabular}{lcc}
\hline Estado Nutricional Aparente & No CASOS & $\%$ \\
\hline Sem registro & 40 & 49,38 \\
Bom & 23 & 28,39 \\
Regular & 13 & 16,04 \\
Ruim & 5 & 6,17 \\
\hline TOTAL & 81 & 100,00 \\
\hline
\end{tabular}

Os dados referentes aos felinos foram distribuídos nas Tabelas de 8 a 10.

TABELA 6 - Distribuiçäo dos sexos de felinos com HNS de 1979 a 1993, no HV da UEL

\begin{tabular}{lcr}
\hline SEXO & No DE CASOS & \multicolumn{1}{c}{$\%$} \\
\hline Sem registro & 2 & 1,471 \\
Macho & 69 & 50,735 \\
Fêmea & 65 & 47,794 \\
\hline
\end{tabular}

TABELA 7 - Distribuiçāo das idades mais frequentes dos felinos com HNS no período de 1979 a 1993 no HV da UEL

\begin{tabular}{lcc}
\hline IDADE (Meses) & No DE CASOS & $\%$ \\
\hline $0-3$ & 51 & 37,5 \\
$3-6$ & 60 & 44,118 \\
$6-9$ & 9 & 6,618 \\
$9-12$ & 2 & 1,47 \\
$12-15$ & 4 & 2,94 \\
Acima de 15 & 3 & 2,206 \\
Sem registro & 7 & 5,147 \\
\hline TOTAL & 136 & 100,00 \\
\hline
\end{tabular}

TABELA 8 - Distribuição das raças dos felinos com HNS no periodo de 1979 a 1993 no HV da UEL

\begin{tabular}{lcc}
\hline RAÇA & No DE CASOS & $\%$ \\
\hline SRD & 73 & 53,676 \\
Siamês & 57 & 41,912 \\
Jaguatirica & 4 & 2,941 \\
Angorá & 1 & 0,735 \\
Persa & 1 & 0,735 \\
\hline TOTAL & 136 & 100,00 \\
\hline
\end{tabular}

TABELA 9 - Distribuição dos tipos de alimentação dos felinos com HNS no periodo de 1979 a 1993 , no HV da UEL

\begin{tabular}{lcc}
\hline TIPO DE ALIMENTAÇÃO & No CASOS & $\%$ \\
\hline Sem registro & 78 & 57,35 \\
Leite & 4 & 2,94 \\
Carne & 13 & 9,55 \\
Ração & 2 & 1,47 \\
Resto de comida & 25 & 18,382 \\
Peixe & 5 & 3,67 \\
Leite e carne & 9 & 6,618 \\
\hline TOTAL & 136 & 100,00
\end{tabular}

TABELA 10 - Distribuição do estado de nutriçāo dos felinos com HNS no periodo de 1979 a 1993, no HV da UEL

\begin{tabular}{lcc}
\hline Estado Nutricional Aparente & No CASOS & $\%$ \\
\hline Sem registro & 82 & 60,29 \\
Bom & 31 & 22,79 \\
Regular & 20 & 14,7 \\
Ruim & 3 & 2,206 \\
\hline TOTAL & 136 & 100,00 \\
\hline
\end{tabular}

\section{DISCUSSÃO E CONCLUSÃO}

O hiperparatireoidismo nutricional secundário uma patologia muito frequente em nosso meio, causando distúrbios no desenvolvimento ósseo de animais em fase de crescimento.

A causa principal desta patologia é a alimentação incorreta de filhotes, como as dietas deficientes em cálcio por exemplo, as que são baseadas no uso de carne bovina, cereais, grãos e frutas. Apesar de termos rações industrializadas balanceadas, muitos animais recebem dietas baseadas quase que exclusivamente em produtos 
cárneos, muito pobres em níveis de cálcio. O leite de vaca, por ser de fácil aquisição, é oferecido ao filhote como fonte adicional de minerais, mas mesmo assim, a dieta do animal não costuma alcançar balanceamento correto, levando ao hiperparatireoidismo nutricional secundário observado por nós e relatados por WATSON, 1981.

As faixas etárias que tiveram as grandes incidências foram do nascimento a seis meses de vida, sendo que deste intervalo a maior ocorrência foi na faixa de 3 a 6 meses, compatível com as observações de BRASS et al. (1975); isto ocorre devido ao grande desenvolvimento corporal e a necessidade de uma alimentação balanceada em cálcio:fósforo, como relatam HUBBARD et al. (1979); MILLER (1969); e HAZEWINKEL (1989).

Neste trabalho verificamos que em relaçao ao sexo, os machos $(59,2 \%$ en cães e $50,7 \%$ em felinos), apresentaram maior incidência do que as fêmeas $(40,7 \%$ em cães e $47,7 \%$ em felinos).

Com relação aos caninos, pode-se observar que as raças de grande porte (Pastor Alemão, Doberman, Fila Brasileiro, Boxer, Dog Alemão, São Bernardo, Akita e Setter), foram as mais atingidas, isto provavelmente deve-se a grande necessidade de cálcio destes animais em sua fase de crescimento e por seu desenvolvimento muito rápido, com uma dieta pobre em cálcio e rica em fósforo, sem a relação Ca:P ideal de 1,2:1,0, concordando com BRASS et al. (1975); GODDARD et al. (1970) e WATSON (1981).

Nos felinos a grande porcentagem de sem raça definida e Siamês, se deve ao número elevado destes ani- mais na região.

Apesar de muitos casos sem registro no tipo de alimentação, se verifica uma dieta rica em fósforo, ou pobre em cálcio, muito distante da relação de cálcio e fósforo ideal na alimentação, assim o alto aporte de fósforo resulta em hiperfosfatemia transitória. Pela lei da saturação iônica, mantendo-se o pH, o produto cálcio e fósforo é uma constante: havendo aumento da fosfatemia, haverá hipocalcemia e subsequente estimulação da paratireóide. Ocorrendo menor disponibilidade de cálcio, há aumento na osteoclasia para sua normatização levando assim a descalcificação óssea, constataçōes verificadas por DOIGE (1990); HAZEWINKEL (1989); GODDAD et al. (1970), PRICE (1970) e KROCK et al. (1968).

Com relação ao maior número de gatos (136) do que de cães (81), observa-se que os felinos são mais seletivos na sua aliṃentação, sendo carnivoros por natureza, já os cães têm o costume de roer ossos, e isto ajuda a diminuir o desbalanceamento de cálcio e fósforo, da mesma forma que os ossos pélvicos e dos membros dos felinos são mais delgados e longos, o que causa fraturas patológicas e maior compressão do canal pélvico levando a casos mais graves de retenção fecal e dificuldade de micção (Figuras 1 e 3).

Quanto a estado de nutrição apesar de os animais observados estarem aparentemente bem nutridos, as interpretações radiogråficas constataram uma descalcificação generalizada dos ossos, com perda da densidade óssea devido ao desbalanceamento, observadas também por HOSKINS (1990) e demonstradas nas Figuras 1 e 2.

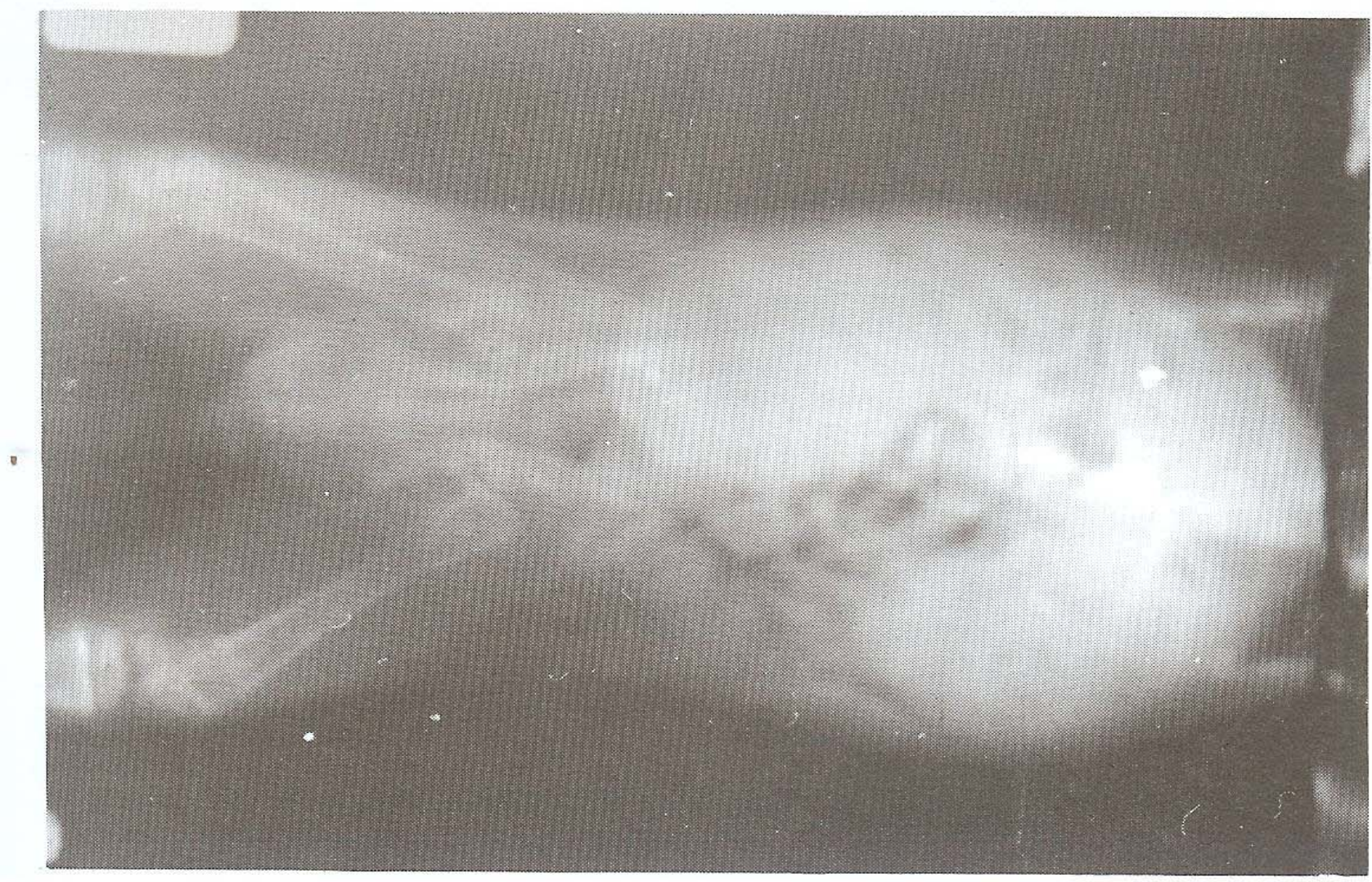

FIG. 1 - Incidência ventro-dorsal, observandọo compressão dos ossos pélvicos com estreitamento do canal pélvico devido a descalcificação 
FIG. 2 - Incidência médio-lateral do membro posterior de cão, observando os ossos descalcificados com cortical fina e transparente com fratura em galho verde na regiāo distal de fêmur

FIG. 3 - Incidência lateral da coluna e abdômen, observando alteraçōes na coluna, devido a descalcificação com lesão evidente em sétima vértebra lombar e osso sacro, com quadro de síndrome de cauda equina, levando a distenção de bexiga 
STURION, Domingos José; PEREIRA, Patrícia Mendes. Radiographic diagnosis of dogs and cats with nutritional secondary hyperparathyroidism. Semina: Ci. Agr., Londrina, v. 16, n. 1, p. 28-33, Mar. 1995.

ABSTRACT: The objective this work was to analyse the radiographic findings executed in the Veterinary Hospital of Universidade Estadual de Londrina from 1978 to 1993 in order to obtain the casuistry of nutritional secondary hiperparathyroidism, trying to observe the distribution related to sex, race, stade of nutrition and type of feeding. A hundred thirty six cats and eighty one dogs, from different races were found in the radiographic findings, the majority belonging the male sex. The most damaged species were those to six months. In the analysis of dogs race we could confirm a great percentage of animals belonging to bog races. The apparent nutrition state was fine for the majority of the animals. The feeding of those animals was defficient in Ca or rich in $P$, leading to an unbalanced diet.

KEY-WORDS: Nutritional secondary hyperparathyroidism; dogs; cats; feeding.

\section{REFERÊNCIAS BIBLIOGRÁFICAS}

BENNETT, D. Nutrition and bone disease in the dog and cat The Veterinary Record, v. 98, p. 310-313, 1976.

BRASS, W.; SCHEBITZ, Ha; DAMMRICH, K. Esqueleto. In: SCHEBITZ, H。; BRASS, W. Cirurgia y patologia quirurgica general veterinåria, 1. ed., Buenos Aires, Editorial Hemisferio Sur S.A. 1979, p. 393-413.

DOIGE, C. Sistema Esquelético. In: THOMPSON, R.G. Patologia Veterinária Especial, São Paulo, Manole, 1990. cap. 11, p. 531-578.

GODDARD, K.M.; WILLIAMS, G.D。; NEWBERNE, P.M. A comparison of all meat, semi-mo ist and dry-type dog foods as diets for growing Beagles. J. Am. Vet Med. Assoc, v. 157, p. 1233, 1970.

HAZEWINKEL, H.A. Nutrition in relation to skeletal growth deformities, Journal of Small Animal Practice, $v_{2}$ 30, n. 11, p. 625-630, 1989 .

HOSKINS, D.J. Nutrition and nutritional disorders. In: VETERINARY PEDIATRICS, DOGS AND CATS FROM BIRTH TO SIX MONTHS. Philadelphia, W.B. Saunders, 1990, cap. 18, p. 473-486.

HUBBARD, G.B。; SCHMIDT, R.E。; GLEISER, C.A。; MACKENZEI, W.F. Effects of hyperparathyroidism and dietary calcium supplementation on bone healing. American Journal of Vet. Research. Aerospace Med. Res, Lab., Wright-Patterson Air Force Base, Dayton, Ohio, v. 40, n. 2, p. 288-293, 1979 .
KROCK, L; BARRET, R.B.; USUI, K. Nutritional secoundary hyperparathyroidism in the cat. Cornell Veto, V. 53, p. 224, 1963.

MILLER, R.M. Nutritional secoundary hyperparathyroidism. Med. Small Anim. Clin., v. 64, p. 400, 1960.

PRICE, D.A. Editorials: Dogs need more than meat J.Am. Veto Med. Assoc., v. 156, p. 681, 1970.

ROWLAND, G.N.; FETTER, A.W. Nutritional secondary hyperparathyroidism. In: BORJRAB, J. Pathophysiology in small an imal surgery, 1. ed. Philadelphia, Lea \& Febiger, 1981, cap. 78, p. 677-680.

ROWLAND, G.No; CAPEN, C.C.; NAGODE, L。A. Experimental hyperparathyroidism in young cats. Pathol. Vet, v. 5, p. 504, 1968.

SCOTT, P.P. Minerals and vitamins in feline nutrition. In: GRAHAM-JONES, O. 1. ed. New York, Pergamon Prees, 1965.

SHER WOOD, L。M॰; MAYER, G.P॰; RAMBERG JR, C.F. Regulation of parathyroid hormone secretion. Proportional control by calcium lack of effect of phosphate. Endocrinology, v. 83, p. 1043, 1968.

WATSON, A.D.J. Nutritional osteodystrophies in dogsa The Veterinary Annual, v. 21, p. 209-219, 1981. 